\title{
O profissional de enfermagem e sua percepção sobre absenteísmo
}

\author{
Jussara Aparecida da Silva Furlan ${ }^{1}$, \\ Katia Stancato ${ }^{2}$, \\ Claudinei José Gomes Campos ${ }^{3}$, \\ Eliete Maria Silva ${ }^{4}$
}

\section{RESUMO}

O absenteísmo na enfermagem é situação de difícil resolução nas organizações de saúde, reconhecida como multifatorial e complexa. Este trabalho analisou a percepção dos profissionais de enfermagem de um Hospital Universitário sobre o absenteísmo. Trata-se de uma pesquisa de natureza qualitativa transversal, norteada por duas questões: “Quais são as consequências do absenteísmo dos profissionais de enfermagem?", e "Que atitudes podem ser adotadas para diminuir o absenteísmo entre os membros da equipe de enfermagem?". Os resultados foram analisados a partir da técnica de análise de conteúdo. Os participantes consideram que o absenteísmo se relaciona com a sobrecarga, o comprometimento da assistência e a perda de benefícios, propondo como estratégias para minimizá-lo: melhores condições de trabalho, motivação e incentivos. Com base nesse estudo, evidencia-se que o absenteísmo constitui um sério fator de imprevisibilidade para a organização, provocado pelo comportamento humano, sendo associado ao clima e a satisfação organizacional.

Descritores: Absenteísmo; Saúde do Trabalhador; Recursos Humanos de Enfermagem; Enfermagem.

\footnotetext{
${ }^{1}$ Enfermeira. Enfermeira do Hospital da Mulher Prof. Dr. José Aristodemo Pinotti da Universidade Estadual de Campinas. Campinas, SP, Brasil. Email: jussara.aps@hotmail.com.

${ }^{2}$ Enfermeira, Doutora em Enfermagem. Professora Doutora da Faculdade de Enfermagem da Universidade Estadual de Campinas. Campinas, SP, Brasil. E-mail: katiast@unicamp.br.

${ }^{3}$ Enfermeiro. Doutor em Ciências Médicas. Professor Doutor da Faculdade de Ciência Médicas da Universidade Estadual de Campinas. Campinas, SP, Brasil. E-mail: ccampos@unicamp.br.

${ }^{4}$ Enfermeira, Doutora em Enfermagem. Professora Associada da Faculdade de Enfermagem da Universidade Estadual de Campinas. Campinas, SP, Brasil. E-mail: emsilva@unicamp.br.
}

Artigo recebido: 11/04/2017.

Artigo aprovado: 03/05/2018.

Artigo publicado: 31/12/2018.

\section{Como citar esse artigo:}

Furlan JAS, Stancato K, Campos CJG, Silva EM O profissional de enfermagem e sua percepção sobre absenteísmo. Rev. Eletr. Enf. [Internet]. 2018 [acesso em: ];20:v20a39. Disponível em: https://doi.org/10.5216/ree.v20.46321. 


\section{INTRODUÇÃO}

No ambiente hospitalar, a Enfermagem é constituída pelo grupo de trabalhadores que se expõe, com frequência, a riscos biológico, físico e psicológico. Esse grupo também é caracterizado por realizar atividades comumente marcadas pela rígida hierarquia, pela divisão de tarefas, pelo cumprimento de protocolos e pelo insuficiente dimensionamento de pessoal, fatores que podem provocar uma alta taxa de absenteísmo(1).

Segundo estudos correntes sobre absenteísmo, num sentido abrangente, este consiste na ausência do trabalhador por um período de dois ou mais dias ${ }^{(2-3)}$. O conceito também pode ser entendido como a soma dos períodos em que os trabalhadores de uma organização não se encontram presentes no trabalho, excluídas as ausências previstas, tais como férias regulares, acidentes de trabalho ou outros motivos que dependam da própria organização(2-3).

Estudo realizado na Croácia analisou os elementos associados ao absenteísmo, afirmando que tal problema representa um verdadeiro desafio para os departamentos de enfermagem, pois podem aumentar os custos, causar eventos adversos à saúde e impactar a qualidade do cuidado prestado ${ }^{(4)}$.

Existem cinco tipos de absenteísmo: absenteísmo-doença (ausência justificada por licença de saúde); absenteísmo por patologia profissional (acidentes de trabalho e/ou doença profissional); absenteísmo legal (amparado por leis, como gestação, nojo, gala, doação de sangue e serviço militar); absenteísmo compulsório (suspensão imposta pelo patrão, por prisão ou por outro impedimento de comparecer ao trabalho); e absenteísmo voluntário (razões particulares não justificadas) ${ }^{(5)}$. Quando relacionado à Enfermagem, o absenteísmo-doença é o mais presente, sendo a principal causa das ausências não previstas, afetando, dessa forma, o andamento laboral, gerando sobrecarga de trabalho para os presentes e prejudicando a qualidade da assistência prestada ${ }^{(6)}$.

Na Noruega e na Dinamarca uma pesquisa explorou as atitudes em relação ao absenteísmo por doença. Pela percepção da enfermagem, o compromisso social com os pacientes influenciou na atitude de ausentar-se ou não. Em outras palavras, neste estudo as atitudes em relação à ausência de doença foram social e moralmente determinadas a nível pessoal, independente do país ${ }^{(7)}$.

Já na França, estudo apontou que há poucas evidências sobre medidas que podem equilibrar os efeitos do absenteísmo. Propõe que se faça um plano para reduzir o absenteísmo na enfermagem, levando em consideração as estratégias utilizadas para amortecer seu impacto, para isso os gerentes devem considerar, por exemplo, a carga de trabalho ou substituição do pessoal ${ }^{(8)}$.

Considerando essa realidade, o presente estudo objetivou estudar a percepção dos profissionais da equipe de enfermagem de um Hospital Universitário sobre o absenteísmo.

As contribuições do estudo podem apoiar o aperfeiçoamento da gestão hospitalar, a melhoria da qualidade de vida dos profissionais e do padrão de atendimento ao paciente. Os resultados podem ainda encorajar reflexões para o alcance de índices menores de absenteísmo nas instituições de saúde.

\section{METODOLOGIA}

Estudo descritivo de natureza qualitativa realizada em 2014 em um Hospital Universitário, de ensino e pesquisa público, de atendimento generalista e de alta complexidade, do interior do Estado de São Paulo (SPBrasil), vinculado ao Sistema Único de Saúde (SUS). A instituição possui capacidade física para 411 leitos de 
internação. Em 2014, o Departamento de Enfermagem contou com 1.550 colaboradores, entre auxiliares e técnicos de enfermagem, enfermeiros e diretores de enfermagem ${ }^{(9)}$.

A população de estudo incluída foi enfermeiros, técnicos e auxiliares de enfermagem que se encontravam acessíveis para responder o questionário durante seu turno de trabalho, incluindo plantão manhã, tarde e noite das diferentes unidades de internação do hospital em estudo. Foram excluídos os trabalhadores da enfermagem que exerciam cargos administrativos, pois optou por estudar o absenteísmo dos trabalhadores que prestam assistência direta ao paciente.

Após autorização do Comitê de Ética em Pesquisa e da superintendência do hospital em estudo, sob o parecer no 1424261, a pesquisadora entregou os questionários nas unidades e após duas horas, estes foram recolhidos. A amostra foi composta por dados de 240 profissionais. O cálculo amostral foi estimado em um trabalho anterior, de cunho quanti-qualitativo, o que justifica o tamanho amostral incomum para pesquisas qualitativas ${ }^{(10)}$.

O questionário continha perguntas de cunho pessoal e duas questões abertas norteadoras deste estudo foram: "Quais são as consequências do absenteísmo dos profissionais de enfermagem?", e "Que atitudes podem ser adotadas para diminuir o absenteísmo entre os membros da equipe de enfermagem?".

Os sujeitos responderam às perguntas de modo a preencher o questionário de próprio punho. Em seguida, procedeu-se à leitura exaustiva das respostas. Por meio desse procedimento, foi possível realizar a construção da grade de análise. Para a análise de conteúdo, foi compreendido como um conjunto de técnicas de pesquisa cujo objetivo é a busca de sentido ou dos sentidos de um documento ${ }^{(11)}$.

Utilizou-se como referencial Bardin ${ }^{(12)}$, respeitando as fases da análise de conteúdo temática: pré-análise, exploração do material, interpretação e inferência. Dessa forma, foi realizada a composição das unidades de análise em categorias, definidas a partir da repetição do conteúdo comum à maioria dos respondentes. Foram identificados os aspectos centrais, que permitiram selecionar 14 unidades de análise de maior relevância para discussão. Cada unidade de análise recebeu uma codificação numérica, correspondente ao número da pergunta e ao número do questionário; por exemplo, no que se refere à pergunta 13 do questionário 192, o código foi 13192.

\section{RESULTADOS E DISCUSSÃO}

Dos entrevistados, $85,95 \%$ eram do sexo feminino, 54,39\% eram casados, $40,77 \%$ trabalhavam no turno da manhã, 43,16\% cursaram até o Ensino Médio, 65,37\% trabalhavam como técnicos de enfermagem, com idade média de 37,03 anos, sendo a idade mínima de 19 anos e a máxima de 67 anos. A média do tempo de serviço na instituição foi de 6,20 anos. Essa média está em conformidade com o trabalho anterior ${ }^{(10)}$. A análise dos dados possibilitou a construção das seguintes categorias temáticas: categorias 1, que permitiram analisar as opiniões que os profissionais de enfermagem atribuem às consequências do absenteísmo; categorias 2, 3 e 4 que contribuíram por analisar, sob a ótica dos profissionais de enfermagem, quais medidas acreditam ser válidas para minimizar o absenteísmo. 


\section{Categoria 1: Sobrecarga de trabalho, comprometimento da assistência ao paciente e perda de benefícios}

No conjunto de respostas, observou-se que os profissionais de Enfermagem participantes do estudo se preocupam com a equipe e com a qualidade da assistência prestada ao paciente, e têm consciência de que os cuidados que o paciente requer são os mesmos, independentemente do número de trabalhadores presentes.

Eles percebem que uma falta compromete o serviço, pois referem que já trabalham em escala reduzida e, no momento que acontece uma ausência, principalmente aquela que não é esperada, o ônus do trabalho aumenta significativamente, pois as atividades deverão ser divididas entre os presentes, conforme explicitado a seguir:

13-092 - Prejuízo para equipe, pois se minha vaga não for reposta na ausência haverá sobrecarga para equipe e prejuízo para a assistência.

13-116 - Quando há falta, os colegas necessitam trabalhar por mim, pois o serviço continua.

13-176 - Como trabalhamos com escala reduzida, uma falta sobrecarrega a equipe toda, prejudicando a assistência aos pacientes.

Para a Enfermagem, as consequências das faltas podem comprometer as ações do cuidado ao paciente, ao estar exigindo, daqueles que estão presentes, agilidade na execução de um número superior de tarefas. 0 absenteísmo de um profissional, não se levando em consideração o motivo, apresenta repercussão imediata, ao reduzir a qualidade da assistência e ao ser capaz de ocasionar o adoecimento do funcionário sobrecarregado ${ }^{(13)}$.

A equipe de enfermagem percebe, ainda, como consequência da falta não justificada a perda de benefícios, como a folga assiduidade, desconto salarial e tempo de serviço para aposentar, e ainda demonstram preocupação em relação à postura assumida diante da chefia, que não poderá mais confiar em seu trabalho.

O trabalho é um gasto de energia ou esforço para um propósito, e esse esforço é parte integral do trabalho do profissional de enfermagem, por isso, esperam ser recompensados por sua contribuição, por meio do salário, de benefícios e de uma boa relação interpessoal. Esse quadro é demonstrado nos excertos seguintes:

13-107 - Desconto no mês, nas férias, no décimo terceiro, na contagem de tempo de serviço, advertência primeira verbal, processo avaliatório.

13-044 -...interfere no prazo da aposentadoria, mal visto pela chefia que não pode contar com você $100 \%$.

13-232 - Desconto em folha quando não tem atestado, perda da folga assiduidade.

O profissional de uma instituição de saúde dedica horas do seu dia ao ofício, em troca da remuneração mensal, o que demonstra, dessa forma, uma permuta entre direitos trabalhistas, compromisso do funcionário e responsabilidades da instituição. A remuneração deve ser adequada e equitativa por suas contribuições e para os objetivos da organização. Caso isso não ocorra, pode vir a gerar insatisfação e o funcionário pode não manter a assiduidade e compromisso com o trabalho ${ }^{(14)}$.

A remuneração nem sempre se dá apenas pelo pagamento do salário. Ela é também, composta pelos benefícios sociais, como férias, décimo terceiro salário, aposentadoria, transporte, vale refeição, gratificações, entre outros. Essas são formas imprescindíveis para manter a satisfação e a produtividade no trabalho ${ }^{(14)}$.

Apesar de a instituição oferecer tais benefícios, o absenteísmo, ainda, constitui um sério fator de incerteza e de imprevisibilidade para a organização, dada a dependência do comportamento humano(15). 
A confiança é associada ao desenvolvimento do clima institucional e ao estímulo à cooperação. Esta promove o fortalecimento das relações internas à organização, desperta a proatividade e favorece o trabalho integrado em equipe ${ }^{(16)}$.

\section{Categoria 2: Melhores condições de trabalho, aumento do quadro de profissionais e redução da jornada de} trabalho

Nesta categoria, destaca-se proposta dos participantes para reduzir o absenteísmo, a respeito de medidas preventivas no ambiente de trabalho. Apresentam como sugestão a readequação do quantitativo do pessoal de enfermagem segundo a complexidade dos pacientes, incremento na boa qualidade de equipamentos e materiais de consumo adquiridos pela instituição, bem como redução da carga horária semanal. Isso pode ser evidenciado nos excertos abaixo:

14-232 - Melhorar as condições de trabalho, como aumento de funcionários, material para trabalho, reconhecimento profissional.

14-192 - Diminuição da carga horária semanal e consciência dos profissionais de seus limites físicos e mental. (Existem colegas com dois a três empregos).

14-143 - Manutenção de escala de serviço adequada, quantidade de profissionais mais adequado à complexidade dos pacientes e serviços.

A equipe de enfermagem, para realizar suas ações, precisa de um local de trabalho saudável, com um dimensionamento de pessoal adequado, com boas condições físicas, contando com materiais e insumos suficientes e em bom estado de conservação, para que o desempenho de suas atividades não seja comprometido ${ }^{(3)}$.

O trabalho na maioria dos ambientes hospitalares é arriscado e insalubre. Em virtude das condições desfavoráveis, os trabalhadores procuram buscar meios para neutralizar sua angústia, posicionando-se defensivamente. Esse meio pode transformá-las em pessoas desinteressadas ao andamento laborativo, escolhendo ausentar-se, através de licenças e atestados médicos ou faltas injustificadas, prejudicando a dinâmica do serviço(2).

Todos esses fatores são acompanhados de dificuldades socioeconômicas enfrentadas pelos profissionais de enfermagem em nosso país, uma vez que recebem baixos salários, favorecendo-os a optar pela dupla jornada de trabalho na necessidade de suprir a família e ter uma vida respeitável. Sabe-se que pessoas que possuem mais de um vínculo empregatício estão impostas a um desgaste físico e emocional maior do que aquelas que optam por um único emprego. No entanto, o salário dos profissionais objeto de estudo é consideravelmente superior ao salário de outras instituições com menor absenteísmo conhecido na região(2,10,17-18).

No período da coleta de dados, o hospital em estudo passava por mobilizações para regulamentação das 30 horas; por esse motivo, a redução da carga horária apareceu em inúmeras respostas, como medida para diminuir o absenteísmo. No seguinte ano, foi estabelecida a nova jornada de trabalho nessa instituição. Este trabalho não se ateve a essa nova configuração, sendo necessário um novo estudo para verificar se houve redução do absenteísmo. 


\section{Categoria 3: Atividades motivacionais, incentivos e flexibilização da escala}

Os sujeitos do estudo apontaram como recomendações para atenuar o absenteísmo a necessidade de a instituição oferecer suporte psicoemocional que propicie atividades motivacionais, que possam ajudar os profissionais a enfrentar a desmotivação no ambiente hospitalar e, também, adequar a vaga de trabalho ao perfil do profissional. Relatam, ainda, a necessidade de receberem incentivos e serem reconhecidos pela chefia, como se pode ver nos trechos abaixo:

14-044 - Reuniões periódicas com as pessoas faltantes com a presença de um psicólogo para tentar motivar e diagnosticar o motivo da falta de motivação. Às vezes o funcionário só recebe cobrança da chefia e nunca reconhecimento pelos acertos. Oferecer incentivos ao funcionário.

14-149 -Acredito na pessoa certa, no lugar certo. A parceria com os profissionais da psicologia para traçar um perfil admissional para os interessados em trabalhar no HC, especificamente.

Os participantes pedem suporte psicológico para motivar a equipe. A desmotivação e a instabilidade no trabalho podem gerar ausências espontâneas, justificadas pela insatisfação e/ou a não valorização do profissional(19).

Uma forma, dentre tantas outras, de satisfazer o profissional, é dar-lhe oportunidade para que se candidate à vaga de trabalho desejada, antes de abrir um recrutamento externo, ou seja, propiciar a transferência de setor na própria instituição. Fonte poderosa de motivação para os empregados, essa oportunidade de remanejamento interno favorece o constante autoaperfeiçoamento e autoavaliação(14).

Outra perspectiva levantada pelos integrantes da pesquisa, está relacionada à desvalorização do profissional com relação à baixa remuneração salarial e a outras formas de benefícios. Os entrevistados indicam que a flexibilização nas trocas de folgas seria importante para prevenir faltas não justificadas. Essa conjuntura é determinada pelo impedimento em oferecer folgas extras ou permitir troca de plantões. Ainda, observou-se um descontentamento em relação aos faltosos que não recebem nenhum tipo de punição pela ausência, conforme evidenciado abaixo:

14-107 - Valorização profissional através de participações em processos avaliatórios e melhores salários, prêmio de incentivo, cursos...

14-080 - Supervisão maleável e compreensivel que possibilite troca de folgas ou banco de horas para eventuais necessidades, atender preferências de folgas etc.

14-092 - Para as faltas injustificadas e recidivas o profissional deve ser avaliado e quando cabivel, advertência, atentar para os faltosos que solicitam hora extra e não comparecem no plantão.

Sabe-se que as pessoas procuram cumprir seus afazeres de forma a receber melhores recompensas e que os incentivos reforçam o aperfeiçoamento. Todas as organizações têm um sistema de incentivos para estimular seus profissionais, e também de punições ou penalidades para coibir certos tipos de comportamentos ${ }^{(14)}$.

Se um indivíduo é admitido, pressupõe-se que ele aceitou as regras da instituição e se comprometeu a seguir as suas normas, em troca de seu pagamento mensal. Em compensação à remuneração, a organização espera que seu funcionário seja eficiente e produtivo, caso contrário, esta não investirá em benefícios para ele ${ }^{(14)}$. 
No entanto, políticas destinadas a aumentar as recompensas por assiduidade (bônus) são inconsistentes, porque estresse e tensões não minimizadas, presentes no ambiente laboral, levam à exaustão física e psicológica do empregado. Logo, o absenteísmo permanece ${ }^{(19)}$.

Os funcionários que não costumam faltar pedem uma chefia mais atenta e a punição dos faltosos. Nesse sentido, os profissionais devem saber qual será a consequência de sua ausência, sendo responsabilidade da organização desenvolver uma política de disciplina progressiva, por exemplo, aplicando advertência oral ou escrita, desconto na folha de pagamento ou até mesmo a demissão do faltoso recorrente. Contudo, estudos mostram que o legislativo ainda não é suficiente para sanar problemas como as faltas excessivas ao longo do ano, justificadas por meio de atestados obtidos em consultas médicas desnecessárias, dificultando, assim, as ações da gerência(14).

Nessa perspectiva, uma das estratégias para reduzir o absenteísmo é, por exemplo, a elaboração de escalas compartilhadas e discutidas com os funcionários, na perspectiva de uma autonomia relativa aliada à responsabilização por um cuidado humanizado(3).

\section{Categoria 4: Compromisso com a equipe e valorização do profissional pela supervisão/gestão}

Os participantes mencionam como medida para minimizar o absenteísmo o fortalecimento da responsabilidade do profissional com a equipe. Consideram, também, o apoio e a valorização do profissional pela chefia como estratégia para a redução das ausências. Apontam que os supervisores e diretores de enfermagem devem reconhecer que os profissionais têm outros afazeres além do trabalho, demonstrando um descontentamento quanto ao relacionamento estabelecido com o dirigente, segundo o que está explicitado pelos discursos abaixo:

14-205 - Maior comprometimento/responsabilidade com o serviço por parte do profissional faltante ...

14-153 - Respeito, chefia ver que também temos vida pessoal fora do trabalho...ajuda entre os colegas do trabalho, reconhecimento do trabalho, valorização.

14-216 - Acredito que quando tem cooperação da chefia temos estímulos para trabalhar... lembrando que muito absenteísmo é falta de vergonha.

O compromisso recíproco é natural dos profissionais que colaboram para um propósito único estabelecido pela equipe. Um dos maiores desafios das organizações é tornar as pessoas comprometidas para atingir as metas estabelecidas coletivamente. Uma forma consiste na transformação através da educação continuada, na qual estabeleça com a equipe a partilha do compromisso com cuidado ao enfermo ${ }^{(14)}$.

As organizações apresentam dificuldades em diferenciar as causas do absenteísmo, por exemplo, quando se trata de falta por doença e/ou falta cometida (não justificável), sendo expresso comumente pela simulação de doença, demostrando, assim, um certo cinismo por parte do trabalhador, fato que pode ser destacado no excerto seguinte: "que muito absenteísmo é falta de vergonha"(20).

Dessa forma, a conciliação dos interesses e das expectativas dos funcionários com os anseios da organização é uma questão complexa. Uma forma de valorizar o profissional é a gestão participativa e democrática, na qual ocorra a valorização das habilidades do profissional que possam contribuir para solução de problemas coletivos ${ }^{(20-21)}$. 
Os sujeitos pesquisados, como forma de atenuar os conflitos internos, propõem reuniões frequentes, por meio do diálogo aberto, em que possam discutir as dificuldades encontradas no ambiente de trabalho, num clima de compreensão, auxílio e confiança no qual estes passam a partilhar a cooperação e regras comuns. Dessa forma, ocorre a instauração de uma ética em que são estabelecidos alicerces do comprometimento recíproco entre os envolvidos ${ }^{(15)}$.

Como limitação do estudo, considera-se que foram utilizadas perguntas de outra pesquisa, sendo que, se houvesse a construção de outro instrumento próprio para essa investigação, outros dados mais aprofundados poderiam ser obtidos. Os excertos não permitiram discriminar as categorias de enfermagem, uma vez que poderiam ocorrer respostas diferentes entre auxiliares, técnicos e enfermeiros.

\section{CONSIDERAÇÕES FINAIS}

Constatou-se que, na percepção dos profissionais de enfermagem, as principais consequências decorrentes do absenteísmo são: a sobrecarga de trabalho, o comprometimento da assistência ao paciente, além da perda de remuneração e benefícios. Foram propostas como medidas para minimizar o absenteísmo: ambiente salubre, redução da jornada de trabalho e aumento do quadro de profissionais, existência de suporte psicoemocional, aumento de incentivos, flexibilização da escala, punição dos faltosos, valorização do profissional, uma equipe comprometida com seu trabalho.

Os resultados desta pesquisa endossam o absenteísmo como um fenômeno corrente na enfermagem e que requer atenção e decisões da gestão dos recursos humanos. Verifica-se a necessidade de estimular o pensamento crítico dos profissionais sobre as ausências. Nota-se que, independentemente da categoria profissional, uma alta taxa de absenteismo desorganiza as atividades da enfermagem e compromete a qualidade da assistência.

Ficou claro pelo levantamento do referencial que o absenteísmo é amplamente estudado de forma quantitativa, trazendo como aspecto singular a esta pesquisa qualitativa, o fenômeno a partir da perspectiva dos profissionais de enfermagem nele envolvidos, considerando os pontos de vista relevantes, apontando que apesar de uma instituição oferecer incentivos aos colaboradores, o absenteísmo, se constitui um sério fator de incerteza e de imprevisibilidade para a organização, provocado pelo comportamento humano, associado ao clima e a satisfação organizacional.

\section{REFERÊNCIAS}

1. Nascimento Júnior FJM, Silva JVF, Rodrigues APRA, Ferreira ALC. A síndrome do desconforto respiratório do recém-nascido: fisiopatologia e desafios assistenciais. Cadernos de Graduação [Internet]. 2014 [cited 2018 Dec 31];2(2):189-98. Disponível em: https://periodicos.set.edu.br/index.php/fitsbiosaude/article/view/1836.

2. Andrade LZC, Silva VM, Lopes MVO, Chaves DBR, Távora RCO. Desobstrução ineficaz de vias aéreas: prevalência e espectro de seus indicadores clínicos. Acta paul. enferm. [Internet]. 2014 [cited 2018 Dec 31];27(4):319-25. Available from:

https://doi.org/10.1590/1982-0194201400054.

3. Herdman TH, Kamitsuru S. NANDA International Nursing Diagnoses: Definitions \& Classification, 2015-2017. Oxford: Wiley Blackwell; 2014.

4. Carvalho OMC, Silva VM, Távora RCO, Chaves DBR, Beltrão BA, Lopes MVO. Adecuación de las características definitorias en los diagnósticos de deterioro del intercambio de gases y de la ventilación espontánea en niños asmáticos. Enfermería Clínica [Internet]. 2015 [cited 2018 Dec 31];25(6):296-304. Available from: https://doi.org/10.1016/j.enfcli.2015.07.007. 
5. Pascoal LM, Lopes MVO, Chaves DBR, Beltrão BA, Silva VM, Monteiro FPM. Impaired gas exchange: accuracy of defining characteristics in children with acute respiratory infection. Rev Lat Am Enfermagem [Internet]. 2015 [cited 2018 Dec 31];23(3):4919. Available from: https://doi.org/10.1590/0104-1169.0269.2581.

6. Pascoal LM, Lopes MV, Silva VM, Beltrão BA, Chaves DB, Santiago JM, et al. Ineffective breathing pattern: defining characteristics in children with acute respiratory infection. Int J Nurs Knowl [Internet]. 2014 [cited 2018 Dec 31];25(1):54-61. Available from: https://doi.org/10.1111/j.2047-3095.2013.01249.x.

7. Pascoal LM, Lopes MVO, Silva VM, Beltrão BA, Chaves DBR, Santiago JMV, et al. Ineffective Breathing Pattern: Defining Characteristics in Children With Acute Respiratory Infection. Int J Nurs Knowl [Internet]. 2014 [cited 2018 Dec 31];25(1):54-61. Available from: https://doi.org/10.1111/j.2047-3095.2013.01249.x.

8. Avena MJ, Pedreira MLG, Herdman TH, Gutiérrez MG. Respiratory Nursing Diagnoses: Presenting Evidence for Identification of the Defining Characteristics in Neonatal and Pediatric Populations. Int J Nurs Knowl [Internet]. 2016 [cited 2018 Dec 31];27(4):184192. Available from: https://doi.org/10.1111/2047-3095.12098.

9. Avena MJ, Pedreira MLG, Gutiérrez MGR. Conceptual validation of the defining characteristics of respiratory nursing diagnoses in neonates. Acta paul. enferm. [Internet]. 2014 [cited 2018 Dec 31];27(1):76-85. Available from: https://doi.org/10.1590/19820194201400015.

10. Avena MJ, Pedreira MLG, Alves LBO, Herdman TH, Gutiérrez MGR. Frequency of Respiratory Nursing Diagnoses and Accuracy of Clinical Indicators in Preterm Infants. Int J Nurs Knowl [Internet]. 2018 [cited 2018 Dec 31]. Available from:

https://doi.org/10.1111/2047-3095.12205.

11. Moraes Filho IM, Souza GB, Nascimento FNN, Santos JLA, Carvalho MR. Checklist do recém-nascido: principais diagnósticos de enfermagem mediante intercorrências e susceptibilidade das mesmas no neonatal. Revista de Divulgação Científica Sena Aires [Internet] 2017 [cited 2018 Dec 31];6(1):30-48. Available from:

http://revistafacesa.senaaires.com.br/index.php/revisa/article/view/276.

12. Carvalho OMC. Medidas de acurácia dos indicadores clínicos dos diagnósticos de enfermagem respiratórios em crianças com asma [Dissertação]. Fortaleza: Programa de Pós-Graduação em Enfermagem/UFC, 2013 [cited 2018 Dec 31 ]. Available from: http://www.repositorio.ufc.br/handle/riufc/8500.

13. Pascoal LM. Validação diferencial dos diagnósticos de enfermagem desobstrução ineficaz de vias aéreas, padrão respiratório ineficaz e troca de gases prejudicada [Tese]. Fortaleza: Programa de Pós-Graduação em Enfermagem/UFC, 2015 [cited 2018 Dec 31]. Available from: http://www.repositorio.ufc.br/handle/riufc/12558.

14. Thomas CM, Kellgren M. Benner's Novice to Expert Model: An Application for Simulation Facilitators. Nurs Sci Q [Internet]. 2017 [cited 2018 Dec 31];30(3):227-234. Available from: https://doi.org/10.1177/0894318417708410.

15. Santos APS, Silva MLC, Souza NL, Mota GM, França DF. Nursing diagnoses of newborns with sepsis in a Neonatal Intensive Care Unit. Rev Lat Am Enfermagem [Internet]. 2014 [cited 2018 Dec 31];22(2):255-61. Available from: https://doi.org/10.1590/01041169.3101.2410.

16. Castro ECM, Leite AJM, Guinsburg R. Mortality in the first 24h of very low birth weight preterm infants in the Northeast of Brazil. Rev Paul Pediatr (English Ed) [Internet]. 2016 [cited 2018 Dec 31];34(1):106-13. Available from:

https://doi.org/10.1016/j.rppede.2015.12.008.

17. Ruschel L, Nader PJH. A doença da membrana hialina em prematuros de baixo peso. Revista da AMRIGS. 2014;58(3):193-7.

18. Pereira JA, Escobar EMA. Cuidados de Enfermagem ao Recém-Nascido Prematuro com Síndrome do Desconforto Respiratório: Revisão Integrativa. Revista Saúde em Foco [Internet]. 2016 [cited 2018 Dec 31];3(2):17-36. Available from:

https://doi.org/10.12819/rsf.2016.3.2.2.

19. Flores BW, Severo GH, Quadros DR, Pisoni L. Assistência de enfermagem ao prematuro com síndrome do desconforto respiratório: uma revisão bibliográfica. Revista Gestão \& Saúde [Internet]. 2017 [cited 2018 Dec 31] 17(1):33-40. Available from: http://www.herrero.com.br/files/revista/file2a2b8c2a12ee96aead66c3bd876cb03e.pdf.

20. Borges JPA. Monitorização da oximetria de pulso em recém-nascidos: atuação do enfermeiro nas unidades neonatais. Revista de Enfermagem e Atenção à Saúde [Internet]. 2013 [cited 2018 Dec 31];2(3):106-14. Available from:

http://seer.uftm.edu.br/revistaeletronica/index.php/enfer/article/view/595.

21. Pascoal LM, Lopes MVO, Silva VM, Chaves DBR, Beltrão BA, Nunes MM, et al. Clinical Differentiation of Respiratory Nursing Diagnoses among Children with Acute Respiratory Infection. J Pediatr Nurs [Internet]. 2016 [cited 2018 Dec 31];31(1):85-91. Available from: https://doi.org/10.1016/j.pedn.2015.08.002. 